\title{
Post - Excisional Defect of Middle Third of Helix Reconstructed by Antia-Buch Helical Chondrocutaneous Advancement Flap
}

Surya Rao Rao Venkata Mahipathy ${ }^{1 *}$, Alagar Raja Durairaj ${ }^{2}$, Narayanamurthy Sundaramurthy ${ }^{3}$, Anand Prasath Jayachandiran $^{4}$, Suresh Rajendran ${ }^{5}$

\footnotetext{
${ }^{1}$ Professor \& Head, Department of Plastic \& Reconstructive Surgery, Saveetha Medical College \& Hospital, Thandalam, Kanchipuram Dist. 602105, Tamilnadu, India

${ }^{2}$ Professor, Department of Plastic \& Reconstructive Surgery, Saveetha Medical College \& Hospital, Thandalam, Kanchipuram Dist. 602105, Tamilnadu, India

${ }^{3}$ Professor, Department of Plastic \& Reconstructive Surgery, Saveetha Medical College \& Hospital, Thandalam, Kanchipuram Dist. 602105, Tamilnadu, India

${ }^{4}$ Assistant Professor \& Head, Department of Plastic \& Reconstructive Surgery, Saveetha Medical College \& Hospital, Thandalam, Kanchipuram Dist. 602105, Tamilnadu, India

${ }^{5}$ Senior Resident, Department of Plastic \& Reconstructive Surgery, Saveetha Medical College \& Hospital, Thandalam, Kanchipuram Dist. 602105, Tamilnadu, India
}

DOI: $10.36347 /$ sasjs.2022.v08i01.011

| Received: 17.12.2021 | Accepted: 25.01.2022 | Published: 29.01.2022

*Corresponding author: Surya Rao Rao Venkata Mahipathy

Professor \& Head, Department of Plastic \& Reconstructive Surgery, Saveetha Medical College \& Hospital, Thandalam, Kanchipuram Dist. 602105, Tamilnadu, India

Abstract

The Antia-Buch flap is a chondrocutaneous helical advancement flap done as a single stage procedure to reconstruct the helix. Here, we present a 62 year old male patient with basal cell carcinoma of the middle $3^{\text {rd }}$ of the helix of the left pinna. He underwent local excision with a $0.5 \mathrm{~cm}$ margin and the resulting defect was reconstructed with an AntiaBuch chondrocutaneous helical advancement flap.

Keywords: Basal Cell Carcinoma, Excision, Helix, Chondrocutaneous Flap.

Copyright $\odot 2022$ The Author(s): This is an open-access article distributed under the terms of the Creative Commons Attribution 4.0 International License (CC BY-NC 4.0) which permits unrestricted use, distribution, and reproduction in any medium for non-commercial use provided the original author and source are credited.

\section{INTRODUCTION}

The mainstay treatment of malignant lesions of the pinna is surgical excision. The reconstruction of the defect following complete excision remains a challenge for the surgeon due to the complex shape of the ear and thin adherent skin. The standard reconstructive options include wedge excision with direct closure, skin grafting, local skin flaps, chondrocutaneous advancement flaps and multistaged reconstruction with tube pedicles. A classic reconstructive technique for chondrocutaneous ear defects is the Antia-Buch flap, which was first described in 1967 [1].

\section{CASE REPORT}

A 62 year old male presented to us with nodular swelling of the left pinna since 1 year. It was spontaneous in onset and gradually progressive. There was no history of pain, ulcaeration or bleeding from the lesion. There was no history of any comorbid illnesses. On examination, there was a $1 \times 0.5 \mathrm{~cm}$ nodular lesion at the middle and lower third junction of the helix, firm in consistency, with minimal induration. There was no regional lymphadenopathy. A clinical diagnosis of basal cell carcinoma was made. We planned for excision with primary repair of the helix. Under local anaesthesia, the lesion was excised with a $0.5 \mathrm{~cm}$ margin and the resultant defect was closed with the Antia-Buch chondrocutaneous helical advancement flap. The postoperative period was uneventful with the flap well settled.

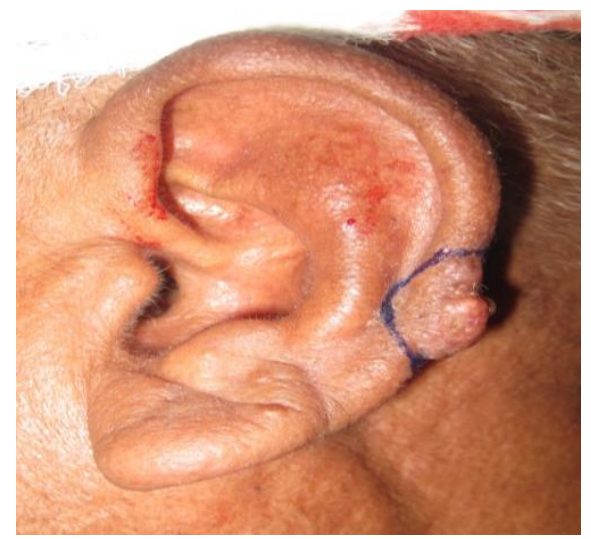

Fig 1: Lesion Lt. Helix 


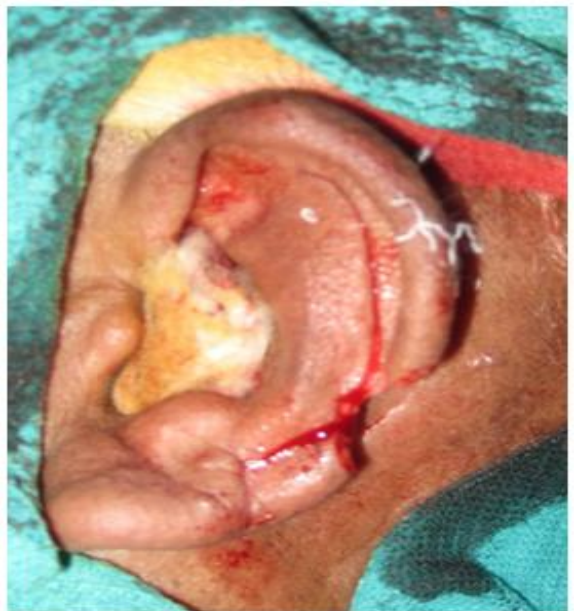

Fig 2: Post excisional defect with incisions for advancement flap

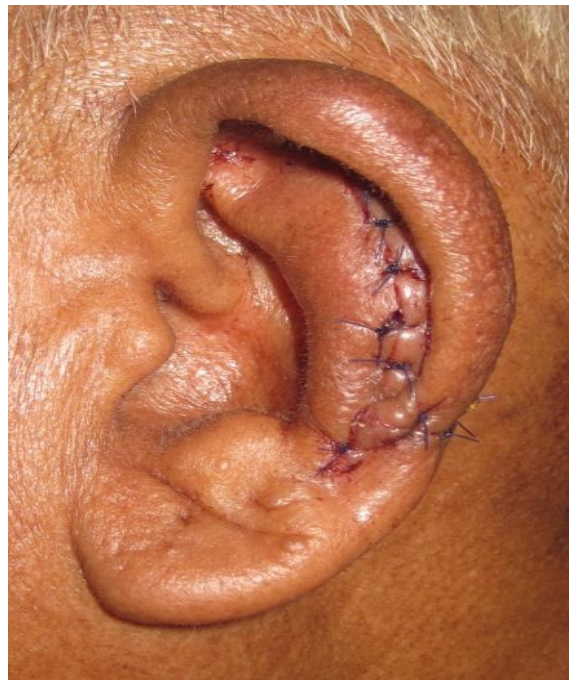

Fig 3: Early post-op picture

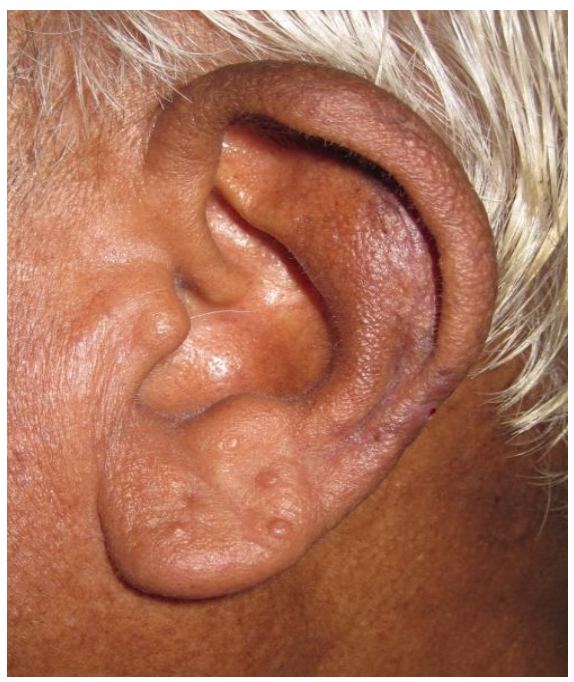

Fig 4: Late post-op picture

\section{DISCUSSION}

More than half of auricular malignancies are situated on the rim of the helix [2]. The external ear defects may be classified according to defect location (superior, middle, or inferior third), thickness (partial or full), and size [small $(<1.5 \mathrm{~cm})$, medium $(1.5-2 \mathrm{~cm})$, or large $(>2 \mathrm{~cm})]$. Small defects can usually be closed directly or with minimal undermining and mobilization of native tissues, or with the use of geometric patterns described by Tanzer designed to approximate skin defects without tension [3]. The auricular repair described by Antia and Buch in 1967 is based on a chondrocutaneous advancement flap suitable for moderate to large sized defects; if larger defects need to be addressed, a V-Y advancement of the helical crus is incorporated into the design with mandatory trimming of the scaphal cartilage to allow a tension-free wound closure $[4,5]$. The ideal reconstruction should be safe, easy to perform, single staged and have a cosmetically acceptable scar with minimal cupping. The chondrocutaneous advancement flap described by Antia and Buch is a good method of reconstructing small to medium sized defects of the helix but when the defect is large (more than $1.5 \mathrm{~cm}$ ), significant cupping occurs. This method relies on bisecting the ear into an anterior skin-cartilage flap and a posterior skin flap. Following this, an incision into the root of the helix is made, to allow ease of advancement of the root of the helix into the defect. To allow tension-free closure, a crescent of healthy cartilage is resected from the scapha, to permit ease of closure of the reconstruction. In this procedure, the anatomical landmarks are restored, whereas scars are concealed in the natural concavities and convexities of the ear [6]. The drawbacks of this technique include extensive bisection of most of the ear, reduction in the ear height due to incorporating the ear lobe into the reconstruction and a visible scar from the incision into the root of the helix needed to allow the advancement of the cephalic chondrocutaneous flap. A modification of this reconstructive method was described by Franssen and Frechner in 2010 where after a wedge of tissue is excised from the ear lobe, the lobe and the ear tissue caudal to the defect are mobilized and incorporated into the reconstruction [7]. This avoids the need to violate the root of the helix and cephalic part of the ear and also avoids the need to excise unaffected scaphal cartilage, thus leaving the ear width unaffected.

\section{CONCLUSION}

The Antia-Buch flap reconstruction is a simple, reliable and convenient technique for small to medium sized defects of the helix that gives a superior aesthetic auricular appearance.

\section{REFERENCES}

1. Antia, N. H., \& Buch, V. I. (1967). Chondrocutaneous advancement flap for the marginal defect of the ear. Plastic and reconstructive surgery, 39(5), 472-477.

2. Blake, G. B., \& Wilson, J. S. P. (1974). Malignant tumours of the ear and their treatment: I. Tumors of the auricle. British journal of plastic surgery, 27(1), 67-76. 
3. Tanzer, R., \& Converse, J. M. (1977). Deformities of the auricle. Reconstructive Plastic Surgery. 2nd ed Philadelphia: WB Saunders

4. Crikelair, G. F. (1956). A method of partial ear reconstruction for avulsion of the upper portion of the ear. Plastic and Reconstructive Surgery, 17(6), 438-443.

5. Thorne, C. H., Beasley, R. W., Aston, S. J., Thorne, C. H., Beasley, R. W., \& Aston, S. J. (2007). Specific regional defects. Grabb and
Smith's Plastic Surgery. Philadelphia, Pa.: Wolters Kluwer Health, 301-304.

6. Low, D. W. (1998). Modified chondrocutaneous advancement flap for ear reconstruction. Plastic and reconstructive surgery, 102(1), 174-177.

7. Franssen, B. B. G. M., \& Frechner, M. R. (2010). Caudal Antia-Buch reconstruction for helical defect reconstruction: Burow's triangle always in the lobule. European Journal of Plastic Surgery, 33(2), 105-107. 